\title{
SOX9: Advances in Gynecological Malignancies
}

\author{
Huan Chen ${ }^{1}$, Yujie $\mathrm{He}^{2}$, Xiangping Wen ${ }^{3}$, Shihong Shao ${ }^{4}$, Yujie Liu ${ }^{1}$ and Jinjin Wang ${ }^{1 *}$ \\ ${ }_{1}^{1}$ Department of Obstetrics and Gynecology, Zhu Zhou Central Hospital, Zhuzhou, China, ${ }^{2}$ Designated Ward, Zhu Zhou \\ Central Hospital, Zhuzhou, China, ${ }^{3}$ Department of Operation, Zhu Zhou Central Hospital, Zhuzhou, China, ${ }^{4}$ Department of \\ Pathology, The Affiliated Hospital of Qingdao University, Qingdao, China
}

\section{OPEN ACCESS}

Edited by:

César López-Camarillo, Universidad Autónoma de la Ciudad de México, Mexico

Reviewed by: Georgios Androutsopoulos, University of Patras, Greece Valerie L. Sodi, Drexel University, United States

*Correspondence: Jinjin Wang wangjinjin412000@163.com

Specialty section:

This article was submitted to Molecular and Cellular Oncology,

a section of the journal

Frontiers in Oncology

Received: 01 September 2021 Accepted: 05 November 2021 Published: 22 November 2021

Citation:

Chen H, He Y, Wen X, Shao S, Liu Y and Wang J (2021) SOX9: Advances in

Gynecological Malignancies.

Front. Oncol. 11:768264.

doi: 10.3389/fonc.2021.768264
Transcription factors of the SOX family were first discovered in mammals in 1990. The sexdetermining region $Y$ box 9 belongs to the SOX transcription factor family. It plays an important role in inducing tissue and cell morphogenesis, survival, and many developmental processes. Furthermore, it has been shown to be an oncogene in many tumors. Gynecological malignancies are tumors that occur in the female reproductive system and seriously threaten the lives of patients. Common gynecological malignancies include ovarian cancer, cervical cancer, and endometrial cancer. So far, the molecular mechanisms related to the incidence and development of gynecological malignancies remain unclear. This makes it particularly important to discover their common causative molecule and thus provide an effective therapeutic target. In recent years, studies have found that multiple mechanisms are involved in regulating the expression of the sexdetermining region $\mathrm{Y}$ box 9 , leading to the occurrence and development of gynecological malignancies. In this review, we discuss the prognostic value of SOX9 expression and the potential of targeting SOX9 for gynecological malignancy treatment. We also discuss progress regarding the role of SOX9 in gynecological malignancy pathogenesis through its mediation of important mechanisms, including tumor initiation and proliferation, apoptosis, migration, invasion, chemoresistance, and stem cell maintenance.

Keywords: SOX9, ovarian cancer, cervical cancer, endometrial cancer, uterine carcinosarcoma

\section{INTRODUCTION}

Transcription factors of the SOX family were first discovered in mammals in 1990. The family is based on the conserved high migration group (HMG) box genes of the mammalian testis determinant Sry. Generally, proteins that contain an HGM domain and have 50\% or higher amino acid similarity to the HMG are called SOX proteins (1). This family is subdivided into eight subgroups, from A-H, each subgroup contains 1-3 members (Table 1) (2). The SOX gene family encodes transcription factors that are conserved across species and participate in important

Abbreviations: OC, ovarian cancer; CC, cervical cancer; EC, endometrial cancer; HGM, high migration group; CSCs, cancer stem cells; ORF, open reading frame; NLS, nuclear localization signal; NES, nuclear output signal; SCST, Sex-cord stromal tumors; SSCTs, Sertoli-Stromal Cell Tumors; GCT, granulosa cell tumors; PFS, Progression-Free-Survival; OS, Overall survival; MCS, multicellular spheroids; OCSCs, ovarian cancer stem cells; EOC, epithelial ovarian cancer; SOC, serous ovarian carcinoma; SNPs, nucleotide polymorphisms; LCR, long control region; EMT, Epithelial mesenchymal transition; EEC, endometrioid carcinoma; USC, uterine serous carcinoma; UCS, Uterine carcinosarcoma. 
TABLE 1 | SOX family and subgroup member.

\begin{tabular}{ll}
\hline Subgroup & \multicolumn{1}{c}{ Member } \\
\hline SoxA & SRY \\
SoxB1 & SOX1, SOX2,SOX3 \\
SoxB2 & SOX14,SOX21 \\
SoxC & SOX4, SOX11 and SOX12 \\
SoxD & SOX5, SOX6 and SOX13, \\
SoxE & SOX8, SOX9 and SOX10 \\
SoxF & SOX7, SOX17 and SOX18 \\
SoxG & SOX15 \\
SoxH & SOX30, \\
\hline SOX famly is subdivdod &
\end{tabular}

SOX family is subdivided into 8 subgroups, from $A-H$, each subgroup contains 1-3 members.SOX9 belongs to SoxE subgroup.

developmental processes (3). The sex-determining region Y box 9 (SOX9) belongs to this family (4). According to the amino acid sequence of the HMG domain and transactivation and dimerization domain, SOX9, SOX8, and SOX10 are grouped into the E subgroup (5). SOX9 protein contains an HMG box DNA binding domain that recognizes (A/T) CAA (T/A) G DNA sequences and controls the expression of target genes (6). It also contains a transcription activation domain located at the $\mathrm{C}$ terminus (7) and plays an important role in inducing tissue and cell morphogenesis, survival (8), and regulation of many developmental processes (9); for example, embryonic development, lineage commitment, and stem cell maintenance (10). SOX9 expression is elevated in numerous types of cancer, including lung, prostate, skin, brain, colorectal, pancreatic, and breast cancer (11-17). These studies indicate that SOX9 acts as an oncogene in many cancers. Several additional studies have shown that SOX9 is involved in the formation of cancer because an increase in its level is conducive to the transformation of stem cells. Furthermore, high levels of SOX9 are related to the tumor grade, poor prognosis, and poor survival of some types of cancer (18). In rodents, the expression of Sry initiates the downstream signal cascade by directly regulating SOX9 (19). However, key SOX9 regulatory genes in most human tissues and cancers have yet to be established; it maybe that they are cell type and developmental stage specific (9). It has been noted that SOX9 has the opposite function of promoting and inhibiting proliferation, which indicates that its functions in proliferation are diverse and vary according to different environments (20). Therefore, the expression and function of SOX 9 alters in different human cancers mainly by regulating the activity of cancer stem cells (CSCs), and as a tumor suppressor under certain circumstances (18).

Gynecological malignancies are tumors that occur in the female reproductive system and seriously threaten the lives of patients (21). Common gynecological malignancies include: ovarian cancer (OC), cervical cancer (CC), and endometrial cancer (EC). To date, the molecular mechanisms related to the incidence and development of gynecological malignancies are still unclear. This makes it particularly pertinent to discover their common causative molecule and to provide an effective therapeutic target. In recent years, studies have found that multiple mechanisms are involved in regulating the expression of the SOX9 gene, leading to the occurrence and development of gynecological malignancies. In this review, we discuss the prognostic value of SOX9 expression and the potential of targeting SOX9 for gynecological malignancy treatment. We also discuss progress regarding the role of SOX9 in gynecological malignancy pathogenesis through its mediation of important mechanisms, including tumor initiation and proliferation, apoptosis, migration, invasion, chemoresistance, and stem cell maintenance.

\section{THE STRUCTURE OF SOX9}

SOX9 is located at $17 \mathrm{q} 24.3 \sim \mathrm{q} 25.1$ in humans. It is 3934 bps in length, has three exons, two introns, and an open reading frame (ORF). Its coding product is a polypeptide containing 509 amino acids (22). A total of 79 amino acids from positions 104 to 182 of the polypeptide chain constitute the HMG box, and it has $71 \%$ homology with the HMG box of the Sry gene (23). Its HMG domain contains two nuclear localization signal (NLS) sequences (24) and one leucine-rich nuclear export signal (NES) sequence (25). These NLS sequences and the NES sequence can be activated by different pathways, enabling SOX9 transcription factors to be present in both the nucleus and cytoplasm, and allowing regulation of the expression of related genes. NLS located at the $\mathrm{N}$-terminus promotes nuclear translocation of SOX9 by binding to calmodulin activated by calcium ions, which can be inhibited by calmodulin specific antagonists (26). NLS located at the $\mathrm{C}$-terminus interacts with importin $\beta$ to form a complex (27), which then, via the RAN GTP-dependent pathway, mediates SOX9 to complete nuclear transport through the nuclear pore (28). Whereas, the NES sequence, located between the two NLS sequences, can interact with chromosome region maintenance 1 to mediate the nuclear export of SOX9 (25). SOX9 is a nuclear protein (24), and it is generally believed that SOX9 nuclear expression has its function (29). The structure and nuclear transport and export mechanisms of SOX9 protein are shown in Figure 1.

\section{OC}

The World Health Organization (WHO) categorizes ovarian tumors according to their histological differentiation, defined as epithelial, sex-cord stromal, and germ cell tumors, of which epithelial ovarian tumors constitute the most common type of OC (30). According to the latest data, OC ranks eighth in the world for female cancers, with 313959 new cases and 207252 deaths in 2020 (31).

\subsection{Sex-Cord Stromal Tumors (SCST)}

SCST account for $10 \%$ of OC, ranging from benign to low-grade malignant, and may be differentiated into male (Sertoli and Leydig cells) or female (granulosa and theca cells) structures. The main types of SCST include granulosa cell tumor, SertoliLeydig cell tumor, and steroid cell tumor (32). 


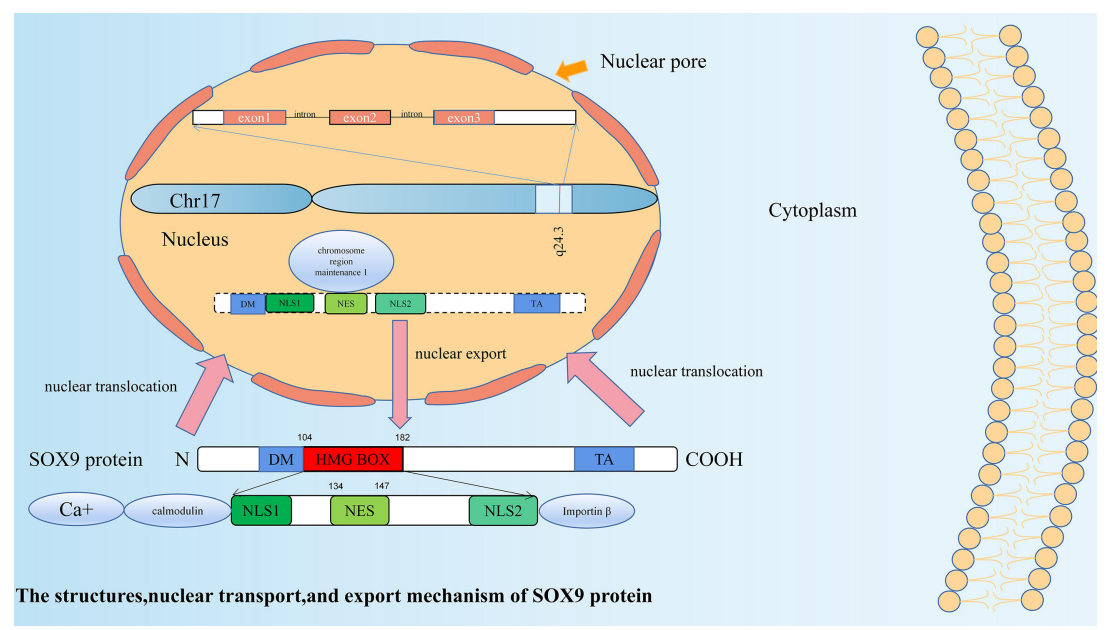

FIGURE 1 | The structure, nuclear transport, and export mechanism of SOX9 protein. SOX9 has three exons, two introns and an open reading frame (ORF). SOX9 protein is a polypeptide containing 509 amino acids. A total of 79 amino acids from positions 104 to 182 of the polypeptide chain constitute the HMG box. In addition to the HMG domain, SOX9 has two other functional domains: a dimerization domain (DIM) and a transactivation domain (TA). Its HMG domain contains two nuclear localization signal (NLS) sequences and one leucine-rich nuclear export signal (NES) sequence. NLS located at the N-terminus promotes nuclear translocation of SOX9 by binding to calmodulin activated by calcium ions. NLS located at the C-terminus interacts with importin $\beta$ to form a complex, which then, via the RAN GTP-dependent pathway, mediates SOX9 to complete nuclear transport through the nuclear pore. The NES sequence located between the two NLS sequences can interact with chromosome region maintenance 1 to mediate the nuclear export of SOX9.

\subsubsection{Sertoli-Stromal Cell Tumors (SSCT)}

SOX9 protein is a downstream effector of the testicular determinant Sry; it plays a core role in Sertoli cell differentiation and testicular cord formation after its translocation to the nucleus. SOX9 is expressed in the testis, particularly in Sertoli cells, while it is inactive in follicular cells of the ovary. Ovarian SSCTs also show testicular differentiation. Therefore, in 2004, Kato et al. examined SOX9 mRNA expression of two SSCT samples. One case was welldifferentiated SSCT and the other was a Sertoli-Leydig tumor of intermediate differentiation. The results showed that SOX9 mRNA was expressed but Sry-specific sequences were not detected in either specimen, while in normal testes and ovaries of the control group, SOX9 was not expressed (33). This suggests that Sryindependent SOX9 expression may be related to Sertoli cell differentiation in SSCT and its potential use as a marker for differential diagnosis. Subsequently, to investigate the role of SOX9 in the histological differential diagnosis of ovarian Sertoli cell tumors from other tumors, Zhao et al. performed SOX9 immunohistochemical staining on 152 ovarian tumors, including 36 pure Sertoli cell tumors, 38 endometrioid borderline tumors, 26 well-differentiated endometrioid carcinomas, 13 sertoliform endometrioid carcinomas, and 39 carcinoid tumors. Results included: (1) A spectrum of immunostaining intensities was shown in all tumor categories, and no significant diagnostic trends were found in any group. (2) The differences in mean immunohistochemical composite scores between the Sertoli cell tumors and the other four tumor categories were not statistically significant. (3) The extent score for sertoliform endometrioid carcinoma showed that SOX9 was found in much lower numbers of cells than other tumor types (34). Therefore, the significance of the expression and function of
SOX9 in ovarian tumors remains unclear. By 2008, Papanastasopoulos et al. had performed immunohistochemical expression of SOX9 through the regulation of prostaglandin D Synthase (Pdgs) in four primary SCST samples, including two Sertoli-Leydig cell tumors (one well-differentiated and one poorly differentiated) and two granulosa-cell tumors. As in the study by Kato et al. (33), SOX9 expression was present in all four specimens and restricted to tumor cells containing a Sertoli-cell component. In contrast, it was not expressed in tumor cells with Leydig-like cell and granulosa-like cell components. The site of expression was mainly cytoplasmic staining, with some nuclei showing positive. $P d g s$ was expressed in both Sertoli-like cell and granulosa-like cell components, but not in Leydig-like cell components (35).

This further demonstrates that Sry-independent SOX9 expression is associated with Sertoli cell differentiation. They also elucidated the mechanism of Sry-independent SOX9 upregulation: $P g d s$ produce prostaglandin D2 (Pgd2), which is necessary and sufficient for recruiting non Sry expressed cells to be able to express SOX9 and differentiate into Sertoli cells.

These studies suggest that SOX9 may be involved in Sertoli cell differentiation in SSCT, and that it is mainly expressed in the cytoplasm; but whether it helps to distinguish OC subtypes remains to be further investigated.

\subsubsection{Granulosa Cell Tumors (GCT)}

In 2010, Kalfa et al. examined SOX9 immunohistochemical expression in six cases of juvenile GCT with hyperandrogenism and 24 cases without hyperandrogenism; they found that there was no correlation between its expression or nuclear localization and hyperandrogenicity (36). In a Foxo1/3 dKO murine model for adult GCT, SOX9 was not present in normal granulosa cells; 
however, it was present in the nuclei of some granulosa cells with follicle-like structures that were apparently missing from the oocytes, as well as in the nuclei of many tumor granulosa cells located within tubular structures. SOX9 expression in mice GCT may reflect more of a shift to an epithelial-like phenotype than a transition to Sertoli-like cells (37).

These findings suggest that SOX9 is predominantly expressed in the nucleus in granulosa cell tumors and may be a potential key to distinguishing this from other subtypes of SCST.

\subsection{Epithelial Tumors}

Ovarian epithelial tumors are the principal pathological type of OC, accounting for $85 \%-90 \%$ of OC (38). SOX9 has been relatively well investigated in this type of tumor. Several studies have found that SOX9 is more highly expressed in OC tissues than in normal ovarian tissues (39-41), adjacent normal counterparts $(42,43)$, or even effusions and solid metastases (44). Therefore, the role of SOX9 in the prognosis of OC patients has been investigated.

\subsubsection{SOX9, Progression-Free-Survival (PFS), and Overall Survival (OS)}

The first study to identify a correlation between SOX9 and prognosis was by Raspaglio et al. There was no association between SOX9 cytoplasmic expression levels and survival outcomes, but OS was significantly shorter in patients with increased nuclear expression of SOX9. The possible mechanism is that SOX9 allows OC cells to survive under hypoxic conditions by activating the expression of $\beta I I I-t u b u l i n$ proteins (45). Subsequently, while studying the role of $S O X 9$ in hepatocellular carcinoma, to examine the prevalence of SOX9 expression as a prognostic factor in other cancers, Richtig et al. analyzed unbiased published datasets for breast, ovarian, lung, and gastric cancers. They found that high SOX9 expression levels were a strong predictor of PFS in OC (46). Similarly to the first study, Sherman-Samis et al. found that higher SOX9 mRNA levels were correlated with shorter OS in a univariate analysis, and a trend towards worse OS was observed for high SOX9 levels in chemotherapy-naïve effusions (44). All these suggest that higher SOX9 levels may be an independent prognostic indicator in ovarian epithelial tumor patients. In the same study, they also found that silencing 70\% of SOX2 and SOX9 significantly reduced the MMP activity of OVCAR3 cells in gelatin-impregnated SDS gels, invasion in the Boyden chamber system in matrix-encapsulated filters, and motility in wound healing assays (44). These findings further suggest that SOX9 may play an important role in the invasive process of ovarian epithelial carcinoma with nuclear expression. Therefore, it could be a potential therapeutic target for OC. The questions remain: what mechanisms are involved in the regulation of SOX9 in OC? and in what ways does its role manifest itself?

\subsubsection{Postranscriptional Regulation of SOX9 Expression by MicroRNAs}

MicroRNAs (miRNAs) are a class of small non-coding RNAs that have important roles in gene regulation (47). In mammals, miRNA regulatory roles have been identified in many areas of biology, which emphasizes that miRNAs are an exciting new class of therapeutic targets with a wide range of applications (48). It was found that $p 70^{S 6 K}$, a key kinase controlling the translation of target mRNAs, directly bind miR-145 and increase the expression of Twist and SOX9, thus promoting the formation of multicellular spheroids (MCS). Whereas, the key to extensive peritoneal dissemination and malignant ascites in OC is the ability to form MCS (49). Dexmedetomidine (DEX), an adjuvant analgesic during cancer treatment, has a dose-dependent suppression effect on OC cells line growth. It upregulates miR-185 expression, which further suppresses SOX9 expression, and then causes inactivation of the Wnt $/ \beta$-catenin signaling pathway, thus inhibiting OC growth and development (50). Xiao et al. found that miR-34c reduced chemoresistance of OC cells to DDP by inhibiting SOX9 expression through the $\beta$-catenin signaling pathway (42). Meanwhile, MiR-30a-5p increased the sensitivity of OC cells to DDP by downregulating SOX9 (41). All these findings suggest that the upregulation of some miRNAs can inhibit SOX9 expression, thereby limiting the growth of OC cells or forming MCS and increasing the sensitivity of OC cells to chemotherapy, and could therefore be used as a more precise treatment target. Similar findings have been found in studies of other members of the SOX family: MiR-138 inhibits OC cell invasion and metastasis by targeting SOX4 and HIF-1 $\alpha$ (51); MiR-223-3p increases OC cell proliferation and invasion by decreasing SOX11 expression (52); MiR-492 promotes migration, invasion, and EMT capabilities via SOX7 in OC (53). These findings reinforce the therapeutic role of the SOX family in tumors.

\subsubsection{Regulation of SOX9 Expression by Long Non-Coding RNAs}

Competing endogenous RNA (ceRNA) networks are increasingly found to play an important role in carcinogenesis (54); it links the function of protein-coding mRNAs to that of non-coding RNAs (55). LncRNAs as endogenous RNA competitive RNAs (ceRNAs) can interact with miRNAs, and miRNAs participate in the regulation of target gene expression by binding to the 3UTR of target mRNAs (56).

To our knowledge, there are only three studies on the regulation of SOX9 by ceRNA network in OC. The first study showed that LINC00115 binds miR-30a then upregulates SOX9 and the $\mathrm{Wnt} / \beta$-catenin pathway, thereby enhancing the stemness of ovarian CSCs and preventing apoptosis (57). The second study showed that LINC01132 acts as an oncogene in epithelial ovarian cancer (EOC) cells by controlling the miR431-5p/SOX9 axis to increase migration and invasion of EOC cells (43). The third study showed that LINC00284 promotes serous ovarian carcinoma (SOC) initiation and progression through the SOX9-LINC00284-miRNA-195/497-5p-mRNA network (40). Taken together, the ceRNA network regulates the expression of SOX9 and plays an important role in ovarian carcinogenesis, development, maintenance of cancer stem cell properties, antiapoptosis, migration, and invasion of cancer cells. These also provide a new direction for the treatment of OC.

\subsubsection{SOX9 and Chemotherapy Resistance}

Chemotherapy resistance is a leading barrier to OC treatment, so overcoming chemoresistance is an important goal in OC treatment 
(58). Studies have shown that SOX9 is involved in this process. SOX9 silencing can slightly sensitize OC cell lines to paclitaxel and cisplatin (DDP) (45). It has also been found that exosome-carried miRNAs regulating SOX9 are involved in the progression of chemotherapy resistance in cancer cells (59). MiR-34c reduces chemoresistance of OC cells to DDP by inhibiting SOX9 expression through the $\beta$-catenin signaling pathway (42). MiR$30 a-5 p$ increases the sensitivity of OC cells to DDP by downregulating SOX9 (41). The results of Shang et al. showed that $S O X 9$, a key super-enhancer regulatory transcription factor (TF) target, is not only required for maintaining the cisplatinresistant state but is also essential for acquiring the cisplatinresistant state in OC cells. There is significant sensitivity to cisplatin in cisplatin-resistant SKOV3 or OVCAR4 cells with depletion of SOX9. The mechanism is likely to involve the binding of SOX9 to the wnt5a loci in the WNT/ $\beta$-catenin pathway (60). These studies suggest that rescuing miRNA expression in OC to inhibit SOX9 and $\mathrm{WNT} / \beta$-catenin signaling activation might provide a promising strategy for dealing with chemoresistance of OC to DDP. The more powerful evidence is the study of Sherman-Samis et al. They found that high mRNA and protein cytoplasmic expression of $S O X 9$ was significantly associated with intrinsic chemoresistance, with a trend of higher expression in patients with poor chemotherapy response to first-line chemotherapy (44). These studies suggest that SOX9 expression can be used as a judgmental indicator prior to chemotherapy selection and also to increase patient sensitivity to chemotherapy by inhibiting its expression.

\subsubsection{Functional Roles of SOX9 in Cancer Stem-Like Cells}

CSCs are some stem-like cells in cancer that have the ability to self-renew and differentiate (61). Characteristics of CSCs include self-renewal, the ability to form spheres and colonies in soft agar, tumor formation in nude mice, and differentiation into stem and non-stem cells in vivo (62). They are thought to be the underlying cause of recurrence, metastasis, and drug resistance in many cancer types (63). Study has identified that SOX9 is a proliferation and stem cell factor in hepatocellular carcinoma (46), and it has the same role in OC. ST6Gal-I induces upregulated expression of key transcription factors such as SOX9 and Slug, which contribute to the growth of spheroids. ST6Gal-I is the main enzyme responsible for nglycan $\alpha 2$ 6sialylation on selected glycoproteins, and its activity regulates cell adhesion, migration, differentiation, and survival (64). By phosphorylating glucose to glucose-6-phosphate (G6P), hexokinases (HKs) catalyze the first irreversible enzymatic step in glucose metabolism. Through 'focal adhesion kinase (FAK)/ extracellular signal-regulated kinase (ERK1/2) activationinduced matrix metalloproteinase 9 (MMP9)/NANOG/SRYBox 9(SOX9),' overexpression of HK2 in OC controls lactate production and promotes metastasis and stemness of OC cells (65). As mentioned previously, LINC00115 is involved in ovarian CSC stemness through the miR30a/SOX9 axis (57).

Most studies suggest that upregulated expression of SOX9 in OC promotes cancer cell proliferation, metastasis, etc. However, there is one study that shows the opposite results. Pgd2 is produced by the enzyme Pgds, which is synthesized in many organs and acts as a signaling molecule in the regulation of various biological processes. It was found that $\operatorname{Pgd} 2$ acts as an autocrine factor to induce SOX9 expression and nuclear translocation. Pgds and SOX9 are highly expressed in proliferatively active solid tumors, while the anti-proliferative effects of $P g d 2 / S O X 9$ are observed in vitro. The possible reason for this may be the induction of apoptotic gene expression owing to the conflict between proliferation and differentiation within tumor cells (39). However, why SOX9 plays the opposite role in vitro study, needs to be further investigated. The regulation mechanism of SOX9 in OC is shown in Figure 2.

\section{CERVICAL CANCER}

CC is one of the most common malignant tumors in female patients. Although early detection of CC improves the prognosis, it is still the fourth most common cause of death in women and ranks first in gynecological malignancies, with 604127 new cases and 41831 deaths in 2020 (31). Therefore, it is necessary to explore the detailed mechanism of CC progression and to identify new effective treatment targets.

\subsection{Evidences for SOX9 Functions as Tumor Suppressor}

While most studies in OC suggest an oncogene role for SOX9 in OC, several studies in CC suggest SOX9 is a suppressor. Methylation of $\mathrm{CpG}$ islands located in gene promoters often leads to transcriptional silencing (66). DNA methylation contributes to various diseases, particularly human cancers (67). The DNA methylation level of SOX9 gradually increases in normal cervical tissues, CIN I, CIN II-III, and CC tissues. This potentially provides a valuable molecular biomarker for CC screening (68). However, the investigators did not assay these specimens for SOX9 expression, therefore, the effect of methylation on the expression of this gene could not be judged. Subsequently, an immunohistochemistry study found that the protein expression of SOX9 decreased gradually in normal cervical tissues, CIN III, and CC tissues. Overexpression of SOX9 could inhibit the proliferation of CC cells in vivo and tumor formation of CC cells in vitro. The possible mechanism is that SOX9 directly transcriptionally activates $\mathrm{p} 21$ by binding to the $\mathrm{p} 21$ promoter sequence of CC cells, then cells arrest at the G1/S phase transition point (69). These findings all suggest that SOX9 is a tumor suppressor gene in CC.

\subsection{SOX9 and Single Nucleotide Polymorphisms (SNP)}

Gene mutations, such as SNP, have been shown to affect cancer susceptibility in recent years (70). Japanese scholars performed genome-wide control studies of five gynecological diseases using data from 46837 subjects ( 5236 fibroids, 645 endometriosis, 647 OC, 909 endometrial cancer, 538 CC, and 39556 shared female controls) from the Japanese biobank project. They found that the 


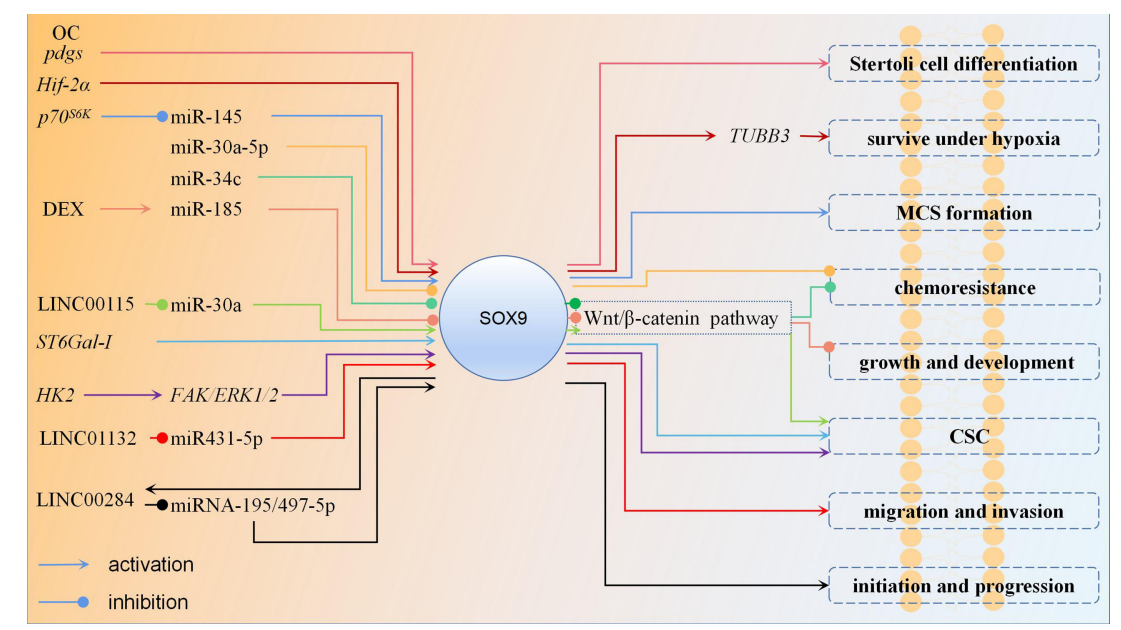

FIGURE 2 | The regulation mechanism of SOX9 in ovarian cancer. In most studies of ovarian cancer, SOX9 plays an oncogene role. Upregulation of SOX9 expression promotes Stertoli cell differentiation, ovarian cancer cell survival under hypoxia, MCS formation, chemoresistance, growth, development, CSC stemness, migration, invasion, initiation and progression. Promoting the expression of certain miRNAs can inhibit the expression of SOX9 and thus suppress ovarian cancer. Wnt/ $\beta$-catenin pathway is also an important pathway, and downregulation of SOX9 expression can inhibit it then suppress ovarian cancer chemoresistance, growth, development, CSC stemness. Each color represent a regulation mechanism of SOX9.

locus rs140991990: $\mathrm{A}>\mathrm{G}$ which is located at SOX9 is associated with the pathogenesis of CC (71). As far as we know, this mutation is located in the intron of SOX9 (https://www.ncbi. nlm.nih.gov/snp/?term=rs140991990). However, intron mutation will only cause changes in the composition and structure of intron transcriptional mRNA, and then affect the maturation and processing of mRNA, finally affecting the protein. The pathogenic risk of SNP in the intron region is significantly lower than that in the gene coding region and regulatory region. Therefore, the role of this SOX9 SNP in CC needs further study.

\subsection{SOX9 and Oncogenes}

Similar to the results of its OC research, the role of SOX9 in CC also has the opposite research results, suggesting that $S O X 9$ plays an oncogene role in CC: (1) Compared with DDP-sensitive tissue, SOX9 was significantly upregulated in DDP-resistant tissues; (2) Knockdown of SOX9 sensitized CC cells to DDP; (3) SOX9 activated the expression of miR-130a by binding to the promoter region of miR-130a, thereby inhibiting the expression of PTEN and CTR1, the downstream genes of miR-130a, and finally promoting the chemoresistance of CC cells to DDP (72); (4) SOX9 was overexpressed in CC in comparison with the control of normal tissues; (5) Downregulation of SOX9 inhibited the growth and metastasis of CC cells, and the upregulation of miR-215-3p inhibited the expression of SOX9, thereby inhibiting the growth and metastasis of CC cells in vivo (73); (6) By inducing SOX9 expression, EGR1 facilitated CC stemness then promoted the proliferation and invasion of CC cells; (7) There was a significant difference in the 5-year OS rate of patients between low and high nucleus expression of SOX9; high SOX9 expression was significantly associated with lower OS rate (74). These studies suggest that upregulation of SOX9 is involved in the formation of CC, chemotherapy resistance, tumor cell stemness, metastasis, and invasion; therefore, high SOX9 expression can be a biomarker for predicting the poor prognosis of CC. The regulation mechanism of SOX9 in CC is shown in Figure 3.

\section{ENDOMETRIAL CANCER}

EC is a malignant tumor of the endometrial epithelium and one of the most common tumors of the female reproductive system (75), with 417367 new cases and 97370 deaths in 2020 (31). It is divided into type I, which is estrogen-related and accounts for $75 \%-90 \%$ of EC, and type II, which is not estrogen-related (76). Type I tumors have a good prognosis and include grade 1-2 EC, while type II malignancies include grade $3 \mathrm{EC}$, uterine serous carcinoma (USC), and clear cell carcinoma, which have a poor prognosis (77). Most EC studies suggest a role for SOX9 as an oncogene. In EC (G1, G2, G3 tumors), atypical hyperplasia and biopsy of normal endometrial specimen tissue (proliferative and secretory stages) samples and nuclear expression of SOX9: (1) was usually found in the epithelial component, but not in the stromal component; (2) was significantly higher in the proliferative phase than in the secretory phase; (3) was significantly higher in the late secretory phase than the early secretory phase; (4) was significantly higher in atypical proliferative lesions than in normal and endometrial cancer tissues; (5) was significantly higher in EC tissue than normal endometrial tissue; (6) was increased significantly and progressively from G1 to G2 to G3 tumors; (7) NF- $\mathrm{KB}$ as well as AKT transcriptionally upregulated SOX9 expression in EC 


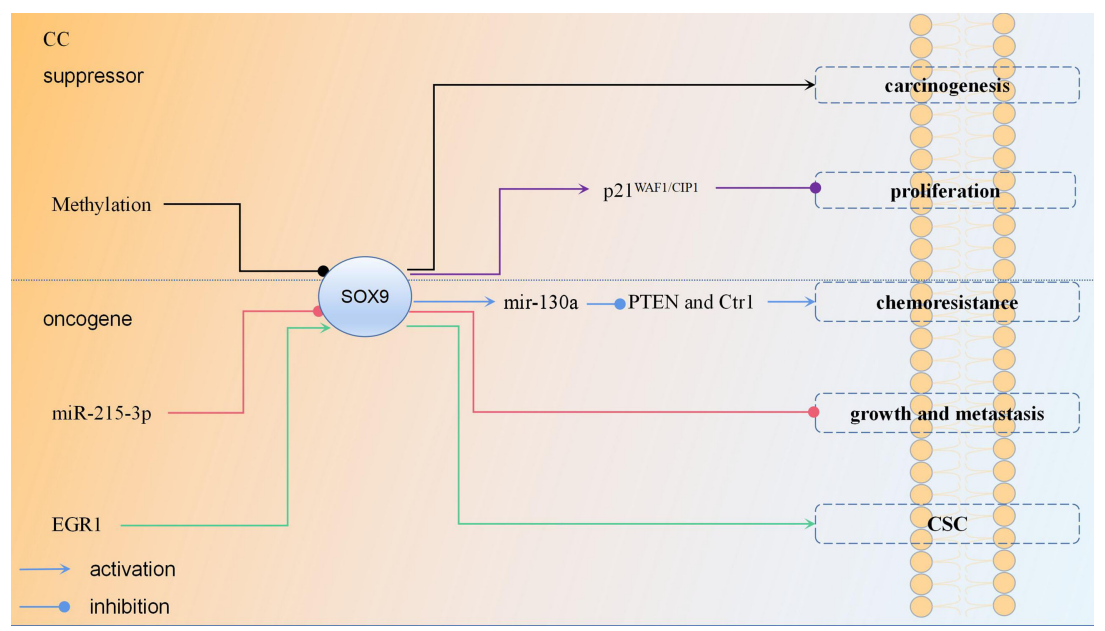

FIGURE 3 | The regulation mechanism of SOX9 in cervical cancer each color represent a regulation mechanism of SOX9.

cells, then activated the $\mathrm{p} 14^{\mathrm{ARF}} / \mathrm{p} 53 / \mathrm{p} 21^{\mathrm{WAF} 1}$ pathway, resulting in inhibition of EC cell proliferation (78). Although these findings suggest an important role for SOX9 in endometrial cell proliferation and carcinogenesis, it cannot explain why the expression of SOX9 in EC is higher than in normal endometrial tissue. Over-expression of SOX9 in a mouse model induced alterations in the tissue structure of the reproductive tract in female mice and played a role in the development of histological lesions similar to endometrial polyps and hyperplasia in humans (79); endometrial hyperplasia is a precancerous lesion of EC (80). This suggests that SOX9 is involved in the pathogenesis of endometrial diseases and may contribute to the formation of EC. SOX9 was significantly more expressed in the UCS than in normal control endometrial tissue in USC of TCGA and GETX datasets. Meanwhile, a 4-gene (KRT23, CXCL1, SOX9, and $A B C A 10)$ signature robustly predicts $O S$ and recurrence-free survival(RFS) of USC (81). Furthermore, the expression of both mRNA and protein of SOX9 was significantly higher in tumor tissues than in paired adjacent tissues. The mechanism was that Circ_0109046 increased SOX9 expression by binding miR-105, which further activated the $\mathrm{Wnt} / \beta$-catenin signaling pathway and promoted EC cell proliferation and metastasis (82). In conclusion, upregulation of SOX9 can promote endometrial cell proliferation, induce endometrial precancerous lesions, and predict the prognosis of EC patients in combination with the expression of other genes.

However, in the study by Opławski et al., the expression of SOX9 was downregulated in endometrioid endometrial adenocarcinomas compared to normal endometrium (83). The possible reason for this result is the different role of SOX9 in different EC subtypes; therefore, these roles need to be further investigated. In the same study, they used sequencing to identify three differentially expressed miRNAs, using the mirTARtool to suggest that miR-144 and miR-30d could target SOX9 (83). This further suggests that miRNA regulation of SOX 9 expression leads to epithelial mesenchymal transition (EMT) development and ultimately to endometrioid adenocarcinogenesis. The regulation mechanism of SOX9 in EC is shown in Figure 4.

\section{UTERINE CARCINOSARCOMA (UCS)}

Uterine carcinosarcoma is a rare gynecological malignancy, accounting for $1 \%-3 \%$ of malignant tumors of the female reproductive tract (84). It is a highly aggressive, biphasic malignancy with carcinomatous and sarcomatous components (85). The former is usually glandular and includes endometrioid, clear cell, or papillary plasmacytoma, while the latter is divided into two categories, homologous (normal uterine tissue resembling endometrial mesenchymal sarcoma, smooth muscle sarcoma or fibrosarcoma) and heterologous (most commonly malignant cartilage or skeletal muscle) (86). UCS with heterologous mesenchymal stroma usually behaves more aggressively and therefore has a worse prognosis than those with homologous features. According to transformation theory, it is believed that the sarcoma component is derived from the carcinoma component through EMT (87). EMT is the process by which epithelial cells transform into an aggressive mesenchymal cell phenotype involved in invasion and metastasis of various cancer types (88). In the study of Yoshida et al., 32 UCSs were investigated. Among them, six patients with non-endometrioid carcinoma showed morphological changes in the heterologous component of the sarcoma component toward the chondrocyte phenotype. It was found that SOX9 expression was significantly different between the two groups, and the mechanism may involve the NF- $\mathrm{KB} / \mathrm{p} 65$ signaling pathway, as well as SOX9, contributing to the change in UCS cell morphology toward the chondrocyte phenotype by regulating COL2A1 transcription (89). However, they did not compare the overall survival and PFS between the two groups. Therefore, the role of $S O X 9$ on their 


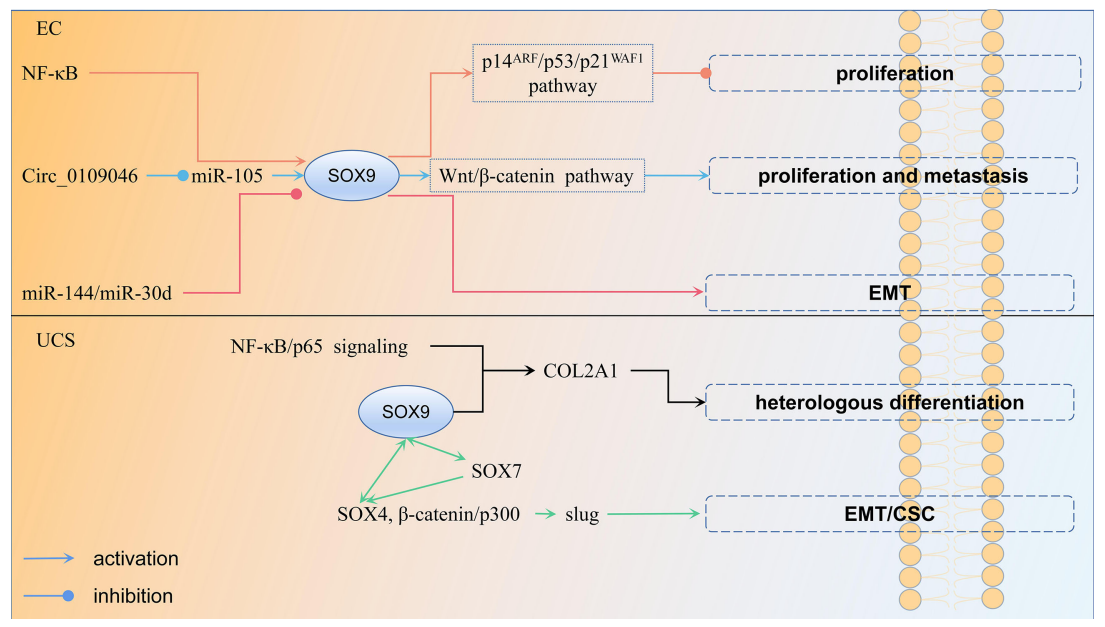

FIGURE 4 | The regulation mechanism of SOX9 in endometrial cancer and uterine carcinosarcoma each color represent a regulation mechanism of SOX9.

TABLE 2 | The expression of SOX9 in gynecological malignancy.

\begin{tabular}{|c|c|c|c|c|c|}
\hline $\begin{array}{l}\text { Types of gynecological } \\
\text { malignancy }\end{array}$ & Subtype & $\begin{array}{l}\text { Expression of } \\
\text { SOX9* }\end{array}$ & Expression site of SOX9 & $\begin{array}{l}\text { Role of } \\
\text { sox9 }\end{array}$ & Referrence \\
\hline \multirow[t]{11}{*}{ OC } & Sertoli-Stromal Cell Tumors & High & Unreported & Oncogene & (33) \\
\hline & $\begin{array}{l}\text { pure Sertoli cell tumor, endometrioid borderline tumor } \\
\text { well-differentiated endometrioid carcinoma sertoliform } \\
\text { endometrioid carcinoma carcinoid tumor }\end{array}$ & No comparison & $\begin{array}{l}\text { Nuclear, cytoplasmic Nuclear, } \\
\text { cytoplasmic, membrane } \\
\text { Nuclear, membrane } \\
\text { Nuclear, cytoplasmic, membrane } \\
\text { Nuclear, cytoplasmic }\end{array}$ & Oncogene & (34) \\
\hline & Sertoli-Stromal Cell Tumors & No comparison & Mainly cytoplasmic, some nuclear & Oncogene & $(35)$ \\
\hline & Juvenile granulosa cell tumors & Unreported & Nuclear & Oncogene & (36) \\
\hline & $\begin{array}{l}\text { serous adenocarcinoma } \\
\text { clear cell adenocarcinoma } \\
\text { Mucinous borderline } \\
\text { dysgerminoma } \\
\text { granulosa cell tumors (GCT), }\end{array}$ & High & Nuclear/cytoplasmic & Oncogene & (39) \\
\hline & serous ovarian carcinoma(SOC) & High & Unreported & Oncogene & $(40)$ \\
\hline & $\begin{array}{l}\text { papillary serous tumors } \\
\text { serous ovarian carcinoma }\end{array}$ & High & Unreported & Oncogene & $(41)$ \\
\hline & Unreported & High & Unreported & Oncogene & $(42)$ \\
\hline & epithelial ovarian cancer(EOC) & High & Unreported & Oncogene & $(43)$ \\
\hline & high-grade serous carcinoma (HGSC). & No comparison & Nuclear/cytoplasmic & Oncogene & (44) \\
\hline & Unreported & No comparison & Nuclear/cytoplasmic & Oncogene & $(45)$ \\
\hline \multirow[t]{3}{*}{ CC } & Unreported & Low & Nuclear & Suppressor & (69) \\
\hline & Unreported & High & Unreported & Oncogene & (73) \\
\hline & Unreported & No comparison & Mainly nuclear & Oncogene & $(74)$ \\
\hline \multirow[t]{3}{*}{ EC } & G1/G2/G3 & High & Nuclear & Oncogene & (81) \\
\hline & uterine serous carcinoma & High & Unreported & Oncogene & $(78)$ \\
\hline & Endometrioid endometrial adenocarcinomas & Low & Unreported & Suppressor & (83) \\
\hline \multirow[t]{4}{*}{ UCS } & endometrioid & No comparison & Nuclear & Oncogene & (89) \\
\hline & Non-endometrioid & & & & \\
\hline & endometrioid & No comparison & Nuclear & Oncogene & (90) \\
\hline & Non-endometrioid & & & & \\
\hline
\end{tabular}

${ }^{*}$ Compared to normal tissue.

prognosis is unknown. SOX9 can also cooperate with other members of its family. Increased expression of SOX7 and SOX9, as well as cooperation between SOX7 and SOX4, are involved in the process in which upregulation of slug by SOX4, $\beta$ - catenin/p300 complexes induces EMT and related CSC properties, which in turn promote homologous and heterologous sarcoma components in UCS (90). In sum, upregulation of SOX9 induces EMT, which leads to the 
TABLE 3 | Studies on non-coding RNA in gynecological malignancy.

\begin{tabular}{|c|c|c|c|c|c|}
\hline Non-coding RNA & Type & Cancer type & sox9 & Fuction & Reference \\
\hline \multirow[t]{7}{*}{ microRNA } & miR-30a-5p up & OC & down & increasing sensitivity to DDP & $(41)$ \\
\hline & miR-34c up & OC & down & reducing chemoresistance & $(42)$ \\
\hline & miR-145 down & OC & up & formation of multicellular spheroids & (49) \\
\hline & miR-185 up & OC & down & inhibiting OC growth and development & (50) \\
\hline & miR-130a up by ${ }^{\star}$ & $\mathrm{CC}$ & up & promoting chemoresistance to DDP & (72) \\
\hline & miR-215-3p up & $\mathrm{CC}$ & down & inhibiting growth and metastasis & (73) \\
\hline & miR-144 up miR-30d down & EC & down & leading to Epithelial mesenchymal transition(EMT) & (83) \\
\hline \multirow[t]{3}{*}{ IncRNA } & LINC00115 binds to miR-30a & OC & up & Enhancing stemness of CSCs and preventing apoptosis & $(57)$ \\
\hline & LINC01132 binds to miR431-5p & OC & up & increasing migration and invasion & (43) \\
\hline & LINC00284-miRNA-195/497-5p-mRNA by* & OC & up & promoting tumor initiation and progression & $(40)$ \\
\hline circular RNAs & Circ_0109046 binding to miR-105 & EC & up & promoting proliferation and metastasis & (82) \\
\hline
\end{tabular}

${ }^{*}$ Represents regulation by SOX9.

SOX9 in different gynecologic tumor cell lines after being regulated by non-coding RNA.

formation of UCS, while contributing to the change of UCS cell morphology to a heterologous mesenchymal stroma, resulting in poor patient prognosis. The regulation mechanism of SOX9 in UCS is shown in Figure 4.

\section{CONCLUSION}

(1) In OC, EC, and UCS, most studies suggest that the expression of SOX9 in cancer tissues is higher than normal control tissues (Table 2) and has a certain relationship with the prognosis. This suggests that it plays an important role in these cancers and may become a new target for treatment. SOX9 is either a tumor suppressor or an oncogene in CC, the current research conclusions are inconclusive, so it remains to be further studied. (2) SOX9 plays an important role in gynecological precancerous lesions, carcinogenesis, development, EMT, chemotherapy resistance, maintenance of cancer stem cell properties, anti-apoptosis, migration, and invasion of cancer cells. (3) There are many mechanisms for regulating SOX9, including epigenetics (methylation of the promoter region); the regulation of non-coding RNA (lncRNA, miRNA, and cirRNA) (Table 3); and several genes. All these provide new ideas for treatment. (4) The WNT/ $\beta$-catenin pathway can be activated by

\section{REFERENCES}

1. Jo A, Denduluri S, Zhang B, Wang Z, Yin L, Yan Z, et al. The Versatile Functions of Sox9 in Development, Stem Cells, and Human Diseases. Genes Dis (2014) 1(2):149-61. doi: 10.1016/j.gendis.2014.09.004

2. Grimm D, Bauer J, Wise P, Krüger M, Simonsen U, Wehland M, et al. The Role of SOX Family Members in Solid Tumours and Metastasis. Semin Cancer Biol (2020) 67(Pt 1):122-53. doi: 10.1016/j.semcancer.2019.03.004

3. Stanisavljevic D, Petrovic I, Vukovic V, Schwirtlich M, Gredic M, Stevanovic $\mathrm{M}$, et al. SOX14 Activates the P53 Signaling Pathway and Induces Apoptosis in a Cervical Carcinoma Cell Line. PloS One (2017) 12(9):e0184686. doi: 10.1371/journal.pone.0184686

4. Yuan X, Li J, Coulouarn C, Lin T, Sulpice L, Bergeat D, et al. SOX9 Expression Decreases Survival of Patients With Intrahepatic Cholangiocarcinoma by Conferring Chemoresistance. Br J Cancer (2018) 119(11):1358-66. doi: $10.1038 / \mathrm{s} 41416-018-0338-9$
SOX9 upregulation to promote carcinogenesis, chemoresistance, cell proliferation, and metastasis. (5) Whether nuclear expression or plasma expression plays a role varies by tumor subtype, but further studies are needed. (6) There is currently no study on the expression of this gene in choriocarcinoma, but the expression of its family genes has been found in germ cell tumors (91) and dissecting gonadoblastoma (92). Both tumors have choriocarcinoma components, so it can be speculated that SOX9 is also expressed in choriocarcinoma; however, confirmation needs further study.

\section{AUTHOR CONTRIBUTIONS}

All authors listed have made a substantial, direct, and intellectual contribution to the work and approved it for publication.

\section{FUNDING}

This work was supported by Effect of neoadjuvant chemotherapy with paclitaxel and carboplatin combined with intraperitoneal perfusion of bevacizumab on prognosis of advanced ovarian cancer [grant numbers S2021SFYLJS0026].

5. Hattori T, Kishino T, Stephen S, Eberspaecher H, Maki S, Takigawa M, et al. E6-AP/UBE3A Protein Acts as a Ubiquitin Ligase Toward SOX9 Protein. J Biol Chem (2013) 288(49):35138-48. doi: 10.1074/jbc.M113.486795

6. Yamashita S, Kataoka K, Yamamoto H, Kato T, Hara S, Yamaguchi K, et al. Comparative Analysis Demonstrates Cell Type-Specific Conservation of SOX9 Targets Between Mouse and Chicken. Sci Rep (2019) 9(1):12560. doi: 10.1038/s41598-019-48979-4

7. Coustry F, Oh CD, Hattori T, Maity SN, de Crombrugghe B, Yasuda H. The Dimerization Domain of SOX9 is Required for Transcription Activation of a Chondrocyte-Specific Chromatin DNA Template. Nucleic Acids Res (2010) 38 (18):6018-28. doi: 10.1093/nar/gkq417

8. Shi G, Sohn KC, Li Z, Choi DK, Park YM, Kim JH, et al. Expression and Functional Role of Sox9 in Human Epidermal Keratinocytes. PloS One (2013) 8(1):e54355. doi: 10.1371/journal.pone. 0054355

9. Ma F, Ye H, He HH, Gerrin SJ, Chen S, Tanenbaum BA, et al. SOX9 Drives WNT Pathway Activation in Prostate Cancer. J Clin Invest (2016) 126 (5):1745-58. doi: 10.1172/JCI78815 
10. Voronkova MA, Luanpitpong S, Rojanasakul LW, Castranova V, Dinu CZ, Riedel H, et al. SOX9 Regulates Cancer Stem-Like Properties and Metastatic Potential of Single-Walled Carbon Nanotube-Exposed Cells. Sci Rep (2017) 7 (1):11653. doi: 10.1038/s41598-017-12037-8

11. Jiang SS, Fang WT, Hou YH, Huang SF, Yen BL, Chang JL, et al. Upregulation of SOX9 in Lung Adenocarcinoma and its Involvement in the Regulation of Cell Growth and Tumorigenicity. Clin Cancer Res (2010) 16(17):4363-73. doi: 10.1158/1078-0432.CCR-10-0138

12. Wang H, Leav I, Ibaragi S, Wegner M, Hu GF, Lu ML, et al. SOX9 is Expressed in Human Fetal Prostate Epithelium and Enhances Prostate Cancer Invasion. Cancer Res (2008) 68(6):1625-30. doi: 10.1158/0008-5472.CAN-07-5915

13. Vidal VP, Ortonne N, Schedl A. SOX9 Expression is a General Marker of Basal Cell Carcinoma and Adnexal-Related Neoplasms. J Cutaneous Pathol (2008) 35(4):373-9. doi: 10.1111/j.1600-0560.2007.00815.x

14. Kordes U, Hagel C. Expression of SOX9 and SOX10 in Central Neuroepithelial Tumor. J Neuro-oncol (2006) 80(2):151-5. doi: 10.1007/ s11060-006-9180-7

15. Matheu A, Collado M, Wise C, Manterola L, Cekaite L, Tye AJ, et al. Oncogenicity of the Developmental Transcription Factor Sox9. Cancer Res (2012) 72(5):1301-15. doi: 10.1158/0008-5472.CAN-11-3660

16. Kopp JL, von Figura G, Mayes E, Liu FF, Dubois CL, Morris JP, et al. Identification of Sox9-Dependent Acinar-to-Ductal Reprogramming as the Principal Mechanism for Initiation of Pancreatic Ductal Adenocarcinoma. Cancer Cell (2012) 22(6):737-50. doi: 10.1016/j.ccr.2012.10.025

17. Jana S, Madhu Krishna B, Singhal J, Horne D, Awasthi S, Salgia R, et al. SOX9: The Master Regulator of Cell Fate in Breast Cancer. Biochem Pharmacol (2020) 174:113789. doi: 10.1016/j.bcp.2019.113789

18. Aldaz P, Otaegi-Ugartemendia M, Saenz-Antoñanzas A, Garcia-Puga M, Moreno-Valladares M, Flores JM, et al. SOX9 Promotes Tumor Progression Through the Axis BMI1-P21cip. Sci Rep (2020) 10(1):357. doi: 10.1038/ s41598-019-57047-w

19. Mamsen LS, Ernst EH, Borup R, Larsen A, Olesen RH, Ernst E, et al. Temporal Expression Pattern of Genes During the Period of Sex Differentiation in Human Embryonic Gonads. Sci Rep (2017) 7(1):15961. doi: 10.1038/s41598-017-15931-3

20. Shi Z, Chiang CI, Labhart P, Zhao Y, Yang J, Mistretta TA, et al. ContextSpecific Role of SOX9 in NF-Y Mediated Gene Regulation in Colorectal Cancer Cells. Nucleic Acids Res (2015) 43(13):6257-69. doi: 10.1093/nar/ gkv568

21. Liu Y, Yi Y, Wu W, Wu K, Zhang W. Bioinformatics Prediction and Analysis of Hub Genes and Pathways of Three Types of Gynecological Cancer. Oncol Lett (2019) 18(1):617-28. doi: 10.3892/ol.2019.10371

22. Vetro A, Ciccone R, Giorda R, Patricelli MG, Della Mina E, Forlino A, et al. XX Males SRY Negative: A Confirmed Cause of Infertility. J Med Genet (2011) 48(10):710-2. doi: 10.1136/jmedgenet-2011-100036

23. Foster JW, Dominguez-Steglich MA, Guioli S, Kwok C, Weller PA, Stevanović M, et al. Campomelic Dysplasia and Autosomal Sex Reversal Caused by Mutations in an SRY-Related Gene. Nature (1994) 372(6506):525-30. doi: $10.1038 / 372525 \mathrm{a} 0$

24. Südbeck P, Scherer G. Two Independent Nuclear Localization Signals are Present in the DNA-Binding High-Mobility Group Domains of SRY and SOX9. J Biol Chem (1997) 272(44):27848-52. doi: 10.1074/jbc.272.44.27848

25. Gasca S, Canizares J, De Santa Barbara P, Mejean C, Poulat F, Berta P, et al. A Nuclear Export Signal Within the High Mobility Group Domain Regulates the Nucleocytoplasmic Translocation of SOX9 During Sexual Determination. Proc Natl Acad Sci USA (2002) 99(17):11199-204. doi: 10.1073/ pnas.172383099

26. Sim H, Rimmer K, Kelly S, Ludbrook LM, Clayton AH, Harley VR. Defective Calmodulin-Mediated Nuclear Transport of the Sex-Determining Region of the Y Chromosome (SRY) in XY Sex Reversal. Mol Endocrinol (2005) 19 (7):1884-92. doi: 10.1210/me.2004-0334

27. Forwood JK, Harley V, Jans DA. The C-Terminal Nuclear Localization Signal of the Sex-Determining Region Y (SRY) High Mobility Group Domain Mediates Nuclear Import Through Importin Beta 1. J Biol Chem (2001) 276 (49):46575-82. doi: 10.1074/jbc.M101668200

28. Stewart M. Structural Biology. Nucl Trafficking Sci (2003) 302(5650):1513-4. doi: 10.1126/science.1092863

29. Xue Y, Lian W, Zhi J, Yang W, Li Q, Guo X, et al. HDAC5-Mediated Deacetylation and Nuclear Localisation of SOX9 is Critical for Tamoxifen
Resistance in Breast Cancer. Br J Cancer (2019) 121(12):1039-49. doi: 10.1038/s41416-019-0625-0

30. Dalezis P, Geromichalou E, Polonifi A, Sagredou S, Nikoleousakos N, Nikolaou M, et al. Azasteroid Alkylators as Dual Inhibitors of AKT and ERK Signaling for the Treatment of Ovarian Carcinoma. Cancers (2020) 12 (5):1263. doi: $10.3390 /$ cancers 12051263

31. Sung H, Ferlay J, Siegel RL, Laversanne M, Soerjomataram I, Jemal A, et al. Global Cancer Statistics 2020: GLOBOCAN Estimates of Incidence and Mortality Worldwide for 36 Cancers in 185 Countries. CA: Cancer J Clin (2021) 71(3):209-49. doi: 10.3322/caac.21660

32. Prat J, Mutch DG. Pathology of Cancers of the Female Genital Tract Including Molecular Pathology. Int J Gynaecol Obstet (2018) 143 Suppl 2:93-108. doi: $10.1002 /$ ijgo. 12617

33. Kato N, Fukase M, Motoyama T. Expression of a Transcription Factor, SOX9, in Sertoli-Stromal Cell Tumors of the Ovary. Int J Gynecol Pathol (2004) 23 (2):180-1. doi: 10.1097/00004347-200404000-00014

34. Zhao C, Bratthauer GL, Barner R, Vang R. Immunohistochemical Analysis of Sox9 in Ovarian Sertoli Cell Tumors and Other Tumors in the Differential Diagnosis. Int J Gynecol Pathol (2007) 26(1):1-9. doi: 10.1097/ 01.pgp.0000232026.22861.b5

35. Papanastasopoulos P, Repanti M, Damaskou V, Bravou V, Papadaki H. Investigating Differentiation Mechanisms of the Constituent Cells of Sex Cord-Stromal Tumours of the Ovary. Virchows Arch (2008) 453(5):465-71. doi: 10.1007/s00428-008-0677-7

36. Kalfa N, Méduri G, Philibert P, Patte C, Boizet-Bonhoure B, Thibaut E, et al. Unusual Virilization in Girls With Juvenile Granulosa Cell Tumors of the Ovary is Related to Intratumoral Aromatase Deficiency. Horm Res Paediatr (2010) 74(2):83-91. doi: 10.1159/000313396

37. Liu Z, Ren YA, Pangas SA, Adams J, Zhou W, Castrillon DH, et al. FOXO1/3 and PTEN Depletion in Granulosa Cells Promotes Ovarian Granulosa Cell Tumor Development. Mol Endocrinol (2015) 29(7):1006-24. doi: 10.1210/ me.2015-1103

38. Chang X, Li D, Liu C, Zhang Z, Wang T. Pentraxin 3 is a Diagnostic and Prognostic Marker for Ovarian Epithelial Cancer Patients Based on Comprehensive Bioinformatics and Experiments. Cancer Cell Int (2021) 21 (1):193. doi: 10.1186/s12935-021-01854-7

39. Malki S, Bibeau F, Notarnicola C, Roques S, Berta P, Poulat F, et al. Expression and Biological Role of the Prostaglandin D Synthase/SOX9 Pathway in Human Ovarian Cancer Cells. Cancer Lett (2007) 255(2):182-93. doi: 10.1016/j.canlet.2007.04.007

40. Wang S, Zhang L, Tao L, Pang L, Fu R, Fu Y, et al. Construction and Investigation of an LINC00284-Associated Regulatory Network in Serous Ovarian Carcinoma. Dis Markers (2020) 2020:9696285. doi: 10.1155/2020/ 9696285

41. Liu R, Zhang Y, Sun P, Wang C. DDP-Resistant Ovarian Cancer CellsDerived Exosomal microRNA-30a-5p Reduces the Resistance of Ovarian Cancer Cells to DDP. Open Biol (2020) 10(4):190173. doi: 10.1098/ rsob.190173

42. Xiao S, Li Y, Pan Q, Ye M, He S, Tian Q, et al. MiR-34c/SOX9 Axis Regulates the Chemoresistance of Ovarian Cancer Cell to Cisplatin-Based Chemotherapy. J Cell Biochem (2019) 120(3):2940-53. doi: 10.1002/jcb.26865

43. Zhu W, Xiao X, Chen J. Silencing of the Long Noncoding RNA LINC01132 Alleviates the Oncogenicity of Epithelial Ovarian Cancer by Regulating the microRNA-431-5p/SOX9 Axis. Int J Mol Med (2021) 48(2):151. doi: 10.3892/ ijmm.2021.4984

44. Sherman-Samis M, Onallah H, Holth A, Reich R, Davidson B. SOX2 and SOX9 are Markers of Clinically Aggressive Disease in Metastatic High-Grade Serous Carcinoma. Gynecol Oncol (2019) 153(3):651-60. doi: 10.1016/ j.ygyno.2019.03.099

45. Raspaglio G, Petrillo M, Martinelli E, Li Puma DD, Mariani M, De Donato M, et al. Sox 9 and Hif- $2 \alpha$ Regulate TUBB3 Gene Expression and Affect Ovarian Cancer Aggressiveness. Gene (2014) 542(2):173-81. doi: 10.1016/ j.gene.2014.03.037

46. Richtig G, Aigelsreiter A, Schwarzenbacher D, Ress AL, Adiprasito JB, Stiegelbauer V, et al. SOX9 is a Proliferation and Stem Cell Factor in Hepatocellular Carcinoma and Possess Widespread Prognostic Significance in Different Cancer Types. PloS One (2017) 12(11):e0187814. doi: 10.1371/ journal.pone.0187814 
47. Zhong X, Pla A, Rayner S. Jasmine: A Java Pipeline for isomiR Characterization in miRNA-Seq Data. Bioinformatics (2019) 36(6):1933-6. doi: 10.1093/bioinformatics/btz806

48. Davis S, Propp S, Freier SM, Jones LE, Serra MJ, Kinberger G, et al. Potent Inhibition of microRNA In Vivo Without Degradation. Nucleic Acids Res (2009) 37(1):70-7. doi: 10.1093/nar/gkn904

49. Lam SS, Ip CK, Mak AS, Wong AS. A Novel P70 S6 kinase-microRNA Biogenesis Axis Mediates Multicellular Spheroid Formation in Ovarian Cancer Progression. Oncotarget (2016) 7(25):38064-77. doi: 10.18632/ oncotarget. 9345

50. Tian H, Hou L, Xiong Y, Cheng Q. Dexmedetomidine Upregulates microRNA-185 to Suppress Ovarian Cancer Growth via Inhibiting the SOX9/Wnt/ß-Catenin Signaling Pathway. Cell Cycle (2021) 20(8):765-80. doi: 10.1080/15384101.2021.1897270

51. Yeh YM, Chuang CM, Chao KC, Wang LH. MicroRNA-138 Suppresses Ovarian Cancer Cell Invasion and Metastasis by Targeting SOX4 and HIF- $1 \alpha$. Int J Cancer (2013) 133(4):867-78. doi: 10.1002/ijc.28086

52. Fang G, Liu J, Wang Q, Huang X, Yang R, Pang Y, et al. MicroRNA-223-3p Regulates Ovarian Cancer Cell Proliferation and Invasion by Targeting SOX11 Expression. Int J Mol Sci (2017) 18(6):1208. doi: 10.3390/ ijms18061208

53. Wang Z, Liu Y, Wang M, Zhao J. Effects of miR-492 on Migration, Invasion, EMT and Prognosis in Ovarian Cancer by Targeting SOX7. J B.U.ON.: Off J Balkan Union Oncol (2020) 25(2):797-804.

54. Ogunwobi OO, Kumar A. Chemoresistance Mediated by ceRNA Networks Associated With the PVT1 Locus. Front Oncol (2019) 9:834. doi: 10.3389/ fonc. 2019.00834

55. Wang QC, Wang ZY, Xu Q, Chen XL, Shi RZ. IncRNA Expression Profiles and Associated ceRNA Network Analyses in Epicardial Adipose Tissue of Patients With Coronary Artery Disease. Sci Rep (2021) 11(1):1567. doi: 10.1038/s41598-021-81038-5

56. Guo Y, Lv B, Liu R, Dai Z, Zhang F, Liang Y, et al. Role of LncRNAs in Regulating Cancer Amino Acid Metabolism. Cancer Cell Int (2021) 21(1):209. doi: 10.1186/s12935-021-01926-8

57. Hou R, Jiang L. LINC00115 Promotes Stemness and Inhibits Apoptosis of Ovarian Cancer Stem Cells by Upregulating SOX9 and Inhibiting the Wnt/ $\beta$ Catenin Pathway Through Competitively Binding to microRNA-30a. Cancer Cell Int (2021) 21(1):360. doi: 10.1186/s12935-021-02019-2

58. Huang HK, Lin YH, Chang HA, Lai YS, Chen YC, Huang SC, et al. Chemoresistant Ovarian Cancer Enhances its Migration Abilities by Increasing Store-Operated Ca2+ Entry-Mediated Turnover of Focal Adhesions. J BioMed Sci (2020) 27(1):36. doi: 10.1186/s12929-020-00630-5

59. Alharbi M, Zuñiga F, Elfeky O, Guanzon D, Lai A, Rice GE, et al. The Potential Role of miRNAs and Exosomes in Chemotherapy in Ovarian Cancer. Endocr Relat Cancer (2018) 25(12):R663-85. doi: 10.1530/ERC-18-0019

60. Shang S, Yang J, Jazaeri AA, Duval AJ, Tufan T, Lopes Fischer N, et al. Chemotherapy-Induced Distal Enhancers Drive Transcriptional Programs to Maintain the Chemoresistant State in Ovarian Cancer. Cancer Res (2019) 79 (18):4599-611. doi: 10.1158/0008-5472.CAN-19-0215

61. Dai Z, Liu P. High Copy Number Variations, Particular Transcription Factors, and Low Immunity Contribute to the Stemness of Prostate Cancer Cells. J Transl Med (2021) 19(1):206. doi: 10.1186/s12967-021-02870-x

62. Basak SK, Veena MS, Oh S, Lai C, Vangala S, Elashoff D, et al. The CD44 (high) Tumorigenic Subsets in Lung Cancer Biospecimens are Enriched for Low miR-34a Expression Published Correction Appears in PLoS One. 2013;8 (9). Doi:10.1371/Annotation/9ae8db84-5d44-4a9c-Bb80-47c9d68820ea. PloS One (2013) 8(9):e73195. doi: 10.1371/journal.pone.0073195

63. Ózsvári B, Magalhães LG, Latimer J, Kangasmetsa J, Sotgia F, Lisanti MP. A Myristoyl Amide Derivative of Doxycycline Potently Targets Cancer Stem Cells (CSCs) and Prevents Spontaneous Metastasis, Without Retaining Antibiotic Activity. Front Oncol (2020) 10:1528. doi: 10.3389/fonc.2020.01528

64. Schultz MJ, Holdbrooks AT, Chakraborty A, Grizzle WE, Landen CN, Buchsbaum DJ, et al. The Tumor-Associated Glycosyltransferase ST6Gal-I Regulates Stem Cell Transcription Factors and Confers a Cancer Stem Cell Phenotype. Cancer Res (2016) 76(13):3978-88. doi: 10.1158/0008-5472.CAN-15-2834

65. Siu M, Jiang YX, Wang JJ, Leung T, Han CY, Tsang BK, et al. Hexokinase 2 Regulates Ovarian Cancer Cell Migration, Invasion and Stemness via FAK/
ERK1/2/MMP9/NANOG/SOX9 Signaling Cascades. Cancers (Basel) (2019) 11(6):813. doi: $10.3390 /$ cancers 11060813

66. Hou Y, Hu J, Zhou L, Liu L, Chen K, Yang X. Integrative Analysis of Methylation and Copy Number Variations of Prostate Adenocarcinoma Based on Weighted Gene Co-Expression Network Analysis. Front Oncol (2021) 11:647253. doi: 10.3389/fonc.2021.647253

67. Choi WL, Mok YG, Huh JH. Application of 5-Methylcytosine DNA Glycosylase to the Quantitative Analysis of DNA Methylation. Int J Mol Sci (2021) 22(3):1072. doi: 10.3390/ijms22031072

68. Wu JH, Liang XA, Wu YM, Li FS, Dai YM. Identification of DNA Methylation of SOX9 in Cervical Cancer Using Methylated-CpG Island Recovery Assay. Oncol Rep (2013) 29(1):125-32. doi: 10.3892/or.2012.2077

69. Wang HY, Lian P, Zheng PS. SOX9, a Potential Tumor Suppressor in Cervical Cancer, Transactivates P21waf1/CIP1 and Suppresses Cervical Tumor Growth. Oncotarget (2015) 6(24):20711-22. doi: 10.18632/oncotarget.4133

70. Peng T, Sun Y, Lv Z, Zhang Z, Su Q, Wu H, et al. Effects of FGFR4 G388R, V10I Polymorphisms on the Likelihood of Cancer. Sci Rep (2021) 11(1):1373. doi: 10.1038/s41598-020-80146-y

71. Masuda T, Low SK, Akiyama M, Hirata M, Ueda Y, Matsuda K, et al. GWAS of Five Gynecologic Diseases and Cross-Trait Analysis in Japanese. Eur J Hum Genet (2020) 28(1):95-107. doi: 10.1038/s41431-019-0495-1

72. Feng C, Ma F, Hu C, Ma JA, Wang J, Zhang Y, et al. SOX9/miR-130a/CTR1 Axis Modulates DDP-Resistance of Cervical Cancer Cell. Cell Cycle (2018) 17 (4):448-58. doi: 10.1080/15384101.2017.1395533

73. Liu CQ, Chen Y, Xie BF, Li YL, Wei YT, Wang F. MicroRNA-215-3p Suppresses the Growth and Metastasis of Cervical Cancer Cell via Targeting SOX9. Eur Rev Med Pharmacol Sci (2019) 23(13):5628-39. doi: 10.26355/ eurrev_201907_18297

74. Zhao J, Li H, Yuan M. EGR1 Promotes Stemness and Predicts a Poor Outcome of Uterine Cervical Cancer by Inducing SOX9 Expression. Genes Genomics (2021) 43(5):459-70. doi: 10.1007/s13258-021-01064-5

75. Cheng Y, Yang L, Shi G, Chen P, Li L, Fang H, et al. Ninjurin 2 Rs 118050317 Gene Polymorphism and Endometrial Cancer Risk. Cancer Cell Int (2021) 21 (1):1. doi: 10.1186/s12935-020-01646-5

76. Chen Q, Tong M, Guo F, Lau S, Zhao M. Parity Correlates With the Timing of Developing Endometrial Cancer, But Not Subtype of Endometrial Cancer. J Cancer (2015) 6(11):1087-92. doi: 10.7150/jca.12736

77. Kwan SY, Au-Yeung CL, Yeung TL, Rynne-Vidal A, Wong KK, Risinger JI, et al. Ubiquitin Carboxyl-Terminal Hydrolase L1 (UCHL1) Promotes Uterine Serous Cancer Cell Proliferation and Cell Cycle Progression. Cancers (Basel) (2020) 12(1):118. doi: 10.3390/cancers12010118

78. Saegusa M, Hashimura M, Suzuki E, Yoshida T, Kuwata T. Transcriptional Up-Regulation of Sox9 by NF-kb in Endometrial Carcinoma Cells, Modulating Cell Proliferation Through Alteration in the P14(ARF)/p53/p21 (WAF1) Pathway. Am J Pathol (2012) 181(2):684-92. doi: 10.1016/ j.ajpath.2012.05.008

79. Gonzalez G, Mehra S, Wang Y, Akiyama H, Behringer RR. Sox9 Overexpression in Uterine Epithelia Induces Endometrial Gland Hyperplasia. Differentiation (2016) 92(4):204-15. doi: 10.1016/ j.diff.2016.05.006

80. Singh S, Pavuluri S, Jyothi Lakshmi B, Biswa BB, Venkatachalam B, Tripura C, et al. Molecular Characterization of Wdr13 Knockout Female Mice Uteri: A Model for Human Endometrial Hyperplasia. Sci Rep (2020) 10(1):14621. doi: 10.1038/s41598-020-70773-w

81. Chen H, Li L, Qin P, Xiong H, Chen R, Zhang M, et al. A 4-Gene Signature Predicts Prognosis of Uterine Serous Carcinoma. BMC Cancer (2021) 21 (1):154. doi: 10.1186/s12885-021-07834-4

82. Li Y, Liu J, Piao J, Ou J, Zhu X. Circ_0109046 Promotes the Malignancy of Endometrial Carcinoma Cells Through the microRNA-105/SOX9/Wnt/ $\beta$ Catenin Axis. IUBMB Life (2021) 73(1):159-76. doi: 10.1002/iub.2415

83. Opławski M, Nowakowski R, Średnicka A, Ochnik D, Grabarek BO, Boroń D. Molecular Landscape of the Epithelial-Mesenchymal Transition in Endometrioid Endometrial Cancer. J Clin Med (2021) 10(7):1520. doi: $10.3390 / \mathrm{jcm} 10071520$

84. Jeong MJ, Park JH, Hur SY, Kim CJ, Nam HS, Lee YS. Preoperative Neutrophil-To-Lymphocyte Ratio as a Prognostic Factor in Uterine Sarcoma. J Clin Med (2020) 9(9):2898. doi: 10.3390/jcm9092898 
85. An Y, Wang Q, Sun F, Zhang G, Wang F, Zhang L, et al. OSucs: An Online Prognostic Biomarker Analysis Tool for Uterine Carcinosarcoma. Genes (Basel) (2020) 11(9):1040. doi: 10.3390/genes11091040

86. McCluggage WG. Malignant Biphasic Uterine Tumours: Carcinosarcomas or Metaplastic Carcinomas? J Clin Pathol (2002) 55(5):321-5. doi: 10.1136/ jcp.55.5.321

87. Tseng JH, Bisogna M, Hoang LN, Olvera N, Rodriguez-Aguayo C, LopezBerestein G, et al. miR-200c-Driven Mesenchymal-To-Epithelial Transition is a Therapeutic Target in Uterine Carcinosarcomas. Sci Rep (2017) 7(1):3614. doi: 10.1038/s41598-017-03972-7

88. Li J, Peng W, Yang P, Chen R, Gu Q, Qian W, et al. MicroRNA-1224-5p Inhibits Metastasis and Epithelial-Mesenchymal Transition in Colorectal Cancer by Targeting SP1-Mediated NF-kb Signaling Pathways. Front Oncol (2020) 10:294. doi: 10.3389/fonc.2020.00294

89. Yoshida T, Hashimura M, Kuwata T, Matsumoto T, Suzuki E, Tazo Y, et al. Transcriptional Regulation of the Alpha-1 Type II Collagen Gene by Nuclear Factor B/p65 and Sox9 in the Chondrocytic Phenotype of Uterine Carcinosarcomas. Hum Pathol (2013) 44(9):1780-8. doi: 10.1016/j.humpath.2012.12.019

90. Inoue $\mathrm{H}$, Takahashi H, Hashimura M, Eshima K, Akiya M, Matsumoto T, et al. Cooperation of Sox 4 With $\beta$-Catenin/P300 Complex in Transcriptional Regulation of the Slug Gene During Divergent Sarcomatous Differentiation in Uterine Carcinosarcoma. BMC Cancer (2016) 16:53. doi: 10.1186/s12885-0162090-y
91. Nonaka D. Differential Expression of SOX2 and SOX17 in Testicular Germ Cell Tumors. Am J Clin Pathol (2009) 131(5):731-6. doi: 10.1309/ AJCP7MNCNBCRN8NO

92. Kao CS, Idrees MT, Young RH, Ulbright TM. "Dissecting Gonadoblastoma" of Scully: A Morphologic Variant That Often Mimics Germinoma. Am J Surg Pathol (2016) 40(10):1417-23. doi: 10.1097/PAS.0000000000000

Conflict of Interest: The authors declare that the research was conducted in the absence of any commercial or financial relationships that could be construed as a potential conflict of interest.

Publisher's Note: All claims expressed in this article are solely those of the authors and do not necessarily represent those of their affiliated organizations, or those of the publisher, the editors and the reviewers. Any product that may be evaluated in this article, or claim that may be made by its manufacturer, is not guaranteed or endorsed by the publisher.

Copyright $(\odot) 2021$ Chen, He, Wen, Shao, Liu and Wang. This is an open-access article distributed under the terms of the Creative Commons Attribution License (CC BY). The use, distribution or reproduction in other forums is permitted, provided the original author(s) and the copyright owner(s) are credited and that the original publication in this journal is cited, in accordance with accepted academic practice. No use, distribution or reproduction is permitted which does not comply with these terms. 